\title{
Direct Parametric Image Reconstruction of Rapid Multi-tracer PET
}

\author{
Xiaoyin Cheng ${ }^{1}$, Nassir Navab ${ }^{2}$, Sibylle I. Ziegler ${ }^{1}$, and Kuangyu Shi ${ }^{1}$ \\ 1 Department of Nuclear Medicine, Technische Universtität München, Germany \\ 2 Computer Aided Medical Procedures, Technische Universtität München, Germany
}

\begin{abstract}
The separation of multiple PET tracers within an overlapped scan based on intrinsic difference of pharmacokinetics is challenging due to the limited SNR of PET measurements and high complexity of fitting models. This study developed a novel direct parametric reconstruction method by integrating a multi-tracer model with reduced number of fitting parameters into image reconstruction. To incorporate the multitracer model, we adopted EM surrogate functions for the optimization of the penalized log-likelihood. The algorithm was validated on realistic simulation phantoms and real rapid $\left[{ }^{18} \mathrm{~F}\right] \mathrm{FDG}$ and $\left[{ }^{18} \mathrm{~F}\right] \mathrm{FLT}$ PET imaging of mice with lymphoma mouse tumor. Both results have been compared with conventional methods and demonstrated evident improvements for the separation of multiple tracers.
\end{abstract}

\section{Introduction}

The complexity of tumor microenvironment reflects multiple physiological features, such as glycolysis, angiogenesis, proliferation, and hypoxia. These properties can be captured by positron-emission tomography (PET) imaging with different radiolabeled metabolites (tracers). The clinical value of combining multiple tracers in oncological detection, staging, localization and the consequent individualization of cancer therapy has been confirmed in several clinical studies [1]. For example, the combination of $\left[{ }^{18} \mathrm{~F}\right] \mathrm{FDG}$ and $\left[{ }^{18} \mathrm{~F}\right] \mathrm{FLT}$ has significantly improved the sensitivity and specificity in the diagnosis of lung nodules [2].

However, radioactive signals of different PET tracers cannot be physically differentiated [3] and the typical practice of multi-tracer PET imaging needs to wait for the full decay and clearance of each tracer, leading to scans in consecutive days. This imposes additional dose due to multiple CT scans on PET/CT as well as increasing labor and financial costs. The possible physiological and anatomical discrepancy due to separate scans may even reduce the expected clinical value. These limitations hamper a wider application of multi-tracer imaging.

Rapid multi-tracer PET imaging aims to differentiate physically identical signals of different tracers based on the intrinsic difference of their pharmacokinetics. This enables the acquisition of multiple tracers with overlaps. In practice, tracers are injected with a short interval of 10-15 mins and activities of different tracers are separated by fitting the superposed pharmacokinetic models of the 
corresponding tracers [45]. However, this method suffers from the low signalto-noise ratio (SNR) of the measured time-activity curves (TACs) and the high complexity of multi-tracer model. The poor quality of model fitting makes the separation of tracers unstable and failure prone. Previous efforts have been made to improve image reconstruction for better kinetic estimation [6], or to reduce the number of fitting parameters for improving stability [17].

One way to improve the pharmacokinetic estimation is to integrate the model fitting into reconstruction [89]. Direct parametric image reconstruction (DPIR) methods utilize the complete spatial and temporal information and highly improves SNR for reconstruction as well as kinetic model fitting. However, conventional DPIR is limited to simple models due to the increased complexity of the objective function for nonlinear model with high parameter dimension.

In this study, we propose a novel DPIR algorithm to reliably separate multitracer signals within a rapid overlapped scan. This is achieved by integrating a multi-tracer model with reduced number of parameters into reconstruction. EM surrogate functions are employed for the optimization of the penalized loglikelihood 1011]. By incorporating the spatiotemporal consistency in model fitting, this method results in reconstructed parametric images with higher SNR and less statistical errors. We evaluated our algorithm both in realistic simulation phantoms and micro-PET scans of mice with lymphoma tumor using $\left[{ }^{18} \mathrm{~F}\right] \mathrm{FDG}$ and $\left[{ }^{18} \mathrm{~F}\right]$ FLT. The results have been compared with conventional indirect methods and demonstrated improved quality for the separation of multiple tracers.

\section{Methods}

\subsection{Multi-tracer Kinetic Model}

A measured TAC $C(t)$ of an image voxel for a dynamic multi-tracer PET scan describes the temporal development of the mixed uptakes of $L$ investigated tracers. It can be modeled as superposition of the $L$ pharmacokinetic models [12]:

$$
\begin{gathered}
\hat{C}(t)=\theta_{B} \bar{C}_{P}+\sum_{l=1}^{L}\left[\theta_{P l} \tilde{C}_{P l}+\theta_{a l} S_{a l}\left(t ; \nu_{a l}, C_{P l}\right)+\theta_{b l} S_{b l}\left(t ; \nu_{a l}, \nu_{b l}, C_{P l}\right)\right] \\
\qquad\left\{\begin{array}{c}
\bar{C}_{P} \triangleq \sum_{l=1}^{L} C_{P l} \\
\tilde{C}_{P l}(t) \triangleq \int_{0}^{t} e^{-(t-\tau)} C_{P l}(\tau) d \tau \\
S_{b l}\left(t ; \nu_{a}, \nu_{b}, C_{P}(t)\right) \triangleq \int_{0}^{t} e^{-\nu_{b l}(t-\tau)}-e^{-\nu_{a l}(t-\tau)} C_{P l}(\tau) d \tau
\end{array}\right.
\end{gathered}
$$

where $\left[\theta_{B}, \theta_{P l}, \theta_{a l}, \theta_{b l}\right]^{T}$ and $\left[\nu_{a l}, \nu_{b l}\right]^{T}$ are intermediate parameters of tracer $l$; $C_{P l}(t)$ is the arterial input function $(\mathrm{AIF})$; Intermediate parameters are combinations of conventional kinetic constants $\mathbf{k}_{l}=\left[K 1_{l}, k 2_{l}, k 3_{l}, k 4_{l}, \theta_{B}\right]^{T} . \theta_{B}$ is the fractional blood volume. The modeled $\hat{C}(t)$ can be expressed as:

$$
\hat{C}(t)=\boldsymbol{\theta}^{T} \mathbf{y}(\boldsymbol{\nu})
$$


where $\boldsymbol{\theta}$ denotes the linear parameters $\left[\theta_{B}, \theta_{P 1}, \theta_{a 1}, \theta_{b 1}, \cdots, \theta_{P L}, \theta_{a L}, \theta_{b L}\right]^{T}$ and y represents the nonlinear parts $\left[\bar{C}_{P}, \tilde{C}_{P 1}, S_{a 1}, S_{b 1}, \cdots, \tilde{C}_{P L}, S_{a L}, S_{b L}\right]^{T}$, which is a function of nonlinear parameters $\boldsymbol{\nu}=\left[\nu_{a 1}, \nu_{b 1}, \cdots, \nu_{a L}, \nu_{b L}\right]$ and AIFs;

Reduced parameter space [7] can be then formulated through calculating the weighted sum square error (WSSE):

$$
W S S E=\sum_{t=1}^{N_{t}} w_{t}\left(C_{t}-\hat{C}_{t}\right)^{2}
$$

where $N_{t}$ is the number of acquisition time points; $w_{t}$ denotes the weights of measurements 12. To minimize Eqn. 4, we set $\partial W S S E / \partial \boldsymbol{\theta}=0$ and derive:

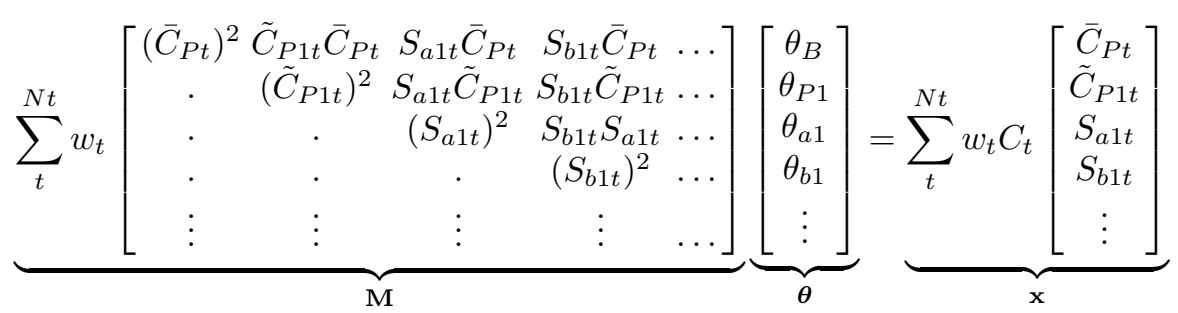

where $\mathbf{M} \in \mathbb{R}^{(3 L+1) \times(3 L+1)}$ is a symmetric matrix.

Substituting the linear parameters $\boldsymbol{\theta}$ in Eqn. 3 with $\mathbf{M}^{-1} \mathbf{x}$, the multi-tracer model $\hat{C}(t)$ can be reduced with only non-linear parameters $\boldsymbol{\nu}$ :

$$
\hat{C}=\left[\mathbf{M}^{-1} \mathbf{x}\right]^{T} \mathbf{y}
$$

Applying Eqn. 6 back in Eqn. 4, $\boldsymbol{\nu}$ can be estimated by minimizing WSSE using numerical algorithms. $\boldsymbol{\theta}$ is calculated analytically and the original kinetic parameters $\mathbf{k}$ can be obtained from $\boldsymbol{\theta}$ and $\boldsymbol{\nu}[12$.

\subsection{Direct Parametric Reconstruction Using Optimization Transfer}

The expectation of projected PET measurements $\mathbf{y}_{k}$ in the $k^{\text {th }}$ frame of sinogram can be expressed as an affine transform of the current image $x_{k}(\boldsymbol{\nu})$ :

$$
\overline{\mathbf{y}}_{k}(\boldsymbol{\nu})=\mathbf{P x}_{k}(\boldsymbol{\nu})+\mathbf{r}_{k}+\mathbf{s}_{k}
$$

$\mathbf{P} \in \mathbb{R}^{N_{i} \times N_{j}}$ is the system matrix where $p_{i, j}$ measures the probability that an event emits from the $j^{t h}$ voxel being detected by the $i^{\text {th }}$ detector pair; $N_{j}$ and $N_{i}$ the total number of image voxels and line-of-responses (LORs); $\mathbf{r}$ and $\mathbf{s}$ are randoms and scatters respectively. The relationship between image intensity $x_{k}\left(\boldsymbol{\nu}_{j}\right)$ and activity concentration $C\left(t ; \boldsymbol{\nu}_{j}\right)$ of voxel $j$ at frame $k$ is given by:

$$
x_{k}\left(\boldsymbol{\nu}_{j}\right)=\int_{t, s}^{t, e} C\left(\tau, \nu_{j}\right) d \tau
$$

where $t, s$ and $t, e$ are the start and end times of frame $k$. 
To estimate the parametric images $\boldsymbol{\nu}$ from $\mathbf{y}$ directly, we are seeking the $\hat{\boldsymbol{\nu}}$ that maximizes the following penalized log-likelihood function $\Phi(\boldsymbol{\nu})$ :

$$
\begin{gathered}
\hat{\boldsymbol{\nu}}=\arg \max _{\boldsymbol{\nu}} \Phi(\boldsymbol{\nu})=\arg \max _{\boldsymbol{\nu}}\{L(\mathbf{y} \mid \boldsymbol{\nu})-\beta U(\boldsymbol{\nu})\} \\
L(\mathbf{y} \mid \boldsymbol{\nu})=\sum_{k=1}^{N_{k}} \sum_{i=1}^{N_{i}} y_{i k} \log \bar{y}_{i k}(\boldsymbol{\nu})-\bar{y}_{i k}(\boldsymbol{\nu})
\end{gathered}
$$

where $L(\mathbf{y} \mid \boldsymbol{\nu})$ is the $\log$-likelihood function with respect to $\boldsymbol{\nu}\left[8 ; N_{k}\right.$ the total number of time frames; $U(\boldsymbol{\nu})$ the smoothness penalty term and $\beta$ the regularization parameter controlling the tradeoff between image resolution and noise. Here a common quadratic penalty is used as described in [11.

To include multi-tracer model Eqn. 6 into reconstruction, maximization of Eqn. 9 is achieved by in each iteration optimizing Expectation Maximization (EM) surrogate functions $Q_{L}, Q_{U}$ designed for $L(\mathbf{y} \mid \boldsymbol{\nu})$ and $U(\boldsymbol{\nu})$ [10 11]:

$$
\left\{\begin{array}{c}
Q_{L}\left(\boldsymbol{\nu} ; \boldsymbol{\nu}^{(n)}\right)=\sum_{j=1}^{N_{j}} \sum_{i=1}^{N_{i}} p_{i j}\left(\sum_{k=1}^{N_{k}} \hat{x}_{j k}^{(n)} \log x_{k}\left(\boldsymbol{\nu}_{j}\right)-x_{k}\left(\boldsymbol{\nu}_{j}\right)\right) \\
Q_{U}\left(\boldsymbol{\nu} ; \boldsymbol{\nu}^{(n)}\right)=\frac{1}{2} \sum_{k=1}^{N_{k}} \sum_{j=1}^{N_{j}} \sum_{l \in \mathcal{N}_{j}} \alpha_{j l}\left(\hat{x}_{j k}^{r e g,(n)}-x_{k}\left(\boldsymbol{\nu}_{j}\right)\right)^{2} \\
\hat{x}_{j k}^{(n)}=\frac{x_{k}\left(\boldsymbol{\nu}_{j}^{(n)}\right)}{\sum_{i=1}^{N_{i}} p_{i j}} \sum_{i=1}^{N i} p_{i j} \frac{y_{i k}}{\bar{y}_{i k}\left(\boldsymbol{\nu}^{(n)}\right)} \\
\hat{x}_{j k}^{r e g,(n)}=\frac{1}{2 \sum_{l \in \mathcal{N}_{j}} \alpha_{j l}} \sum_{l \in \mathcal{N}_{j}} \alpha_{j l}\left(x_{k}\left(\boldsymbol{\nu}_{j}^{(n)}\right)+x_{k}\left(\boldsymbol{\nu}_{l}^{(n)}\right)\right)
\end{array}\right.
$$

where $\mathcal{N}_{j}$ is the set of neighborhood voxels centering around voxel $j ; \alpha_{j l}$ the weighting factors set to be the inverse of distance between voxels $j$ and $l ; \hat{\mathbf{x}}^{(n)}$ and $\hat{\mathbf{x}}^{r e g,(n)}$ are intermediate reconstructed images and smoothed images respectively at the $n^{\text {th }}$ iteration.

The overall surrogate function is then $Q \triangleq Q_{L}-\beta Q_{U}$. Thus the maximization of Eqn.9 is transferred to maximize $Q\left(\boldsymbol{\nu} ; \boldsymbol{\nu}^{(n)}\right)$. As both $Q_{L}$ and $Q_{U}$ are separable for voxels, the maximization can be further transferred into a voxel-wise scale:

$$
\boldsymbol{\nu}_{j}^{(n+1)}=\arg \max _{\boldsymbol{\nu}_{j}} q\left(\boldsymbol{\nu}_{j} ; \boldsymbol{\nu}_{j}^{(n)}\right)=\arg \max _{\boldsymbol{\nu}_{j}} q_{L}\left(\boldsymbol{\nu}_{j} ; \boldsymbol{\nu}_{j}^{(n)}\right)-\beta q_{U}\left(\boldsymbol{\nu}_{j} ; \boldsymbol{\nu}_{j}^{(n)}\right)
$$

Eqn. 12 resembles a 1D curve fitting problem. Here we used the modified Levenberg-Marquardt (LM) algorithm as described in [1] and combined with the Hooke and Jeeves pattern search.

Overall, the proposed algorithm consists of three main steps. Given $\boldsymbol{\nu}^{(0)}$, the dynamic dual-tracer images $\mathbf{x}$ is initialized in Eqn. 8, Each iteration consists of a frame-wise reconstruction to obtain $Q_{L}$, a frame-wise regularization to calculate $Q_{U}$ in case of $\beta>0$, and a voxel-wise fitting step to update $\boldsymbol{\nu}$. As loops end, linear parameters $\boldsymbol{\theta}$ are calculated from Eqn. [5] analytically. Separated dynamic images of each tracer are retrieved from corresponding pharmacokinetic models. 


\section{$3 \quad$ Results}

To validate the algorithm, a realistic phantom was generated using computational simulations following the procedure in [11. The combination of $\left[{ }^{18} \mathrm{~F}\right] \mathrm{FDG}$ and $\left[{ }^{18} \mathrm{~F}\right] \mathrm{FET}$ were simulated with an interval of $15 \mathrm{mins}$ for 45 minutes of 65 frames on the Zubal brain phantom, which includes gray matter (2 types), white matter, caudate, putamen, skin, skull, thalamus and an additional tumor. The pharmacokinetic parameters of $\left[{ }^{18} \mathrm{~F}\right] \mathrm{FDG}$ and $\left[{ }^{18} \mathrm{~F}\right] \mathrm{FET}$ were derived from clinic data. To test the separation of tracers, exclusive uptake was assumed on the tumor area and therefore there is only contrast in FET and the tumor is not differentiable in FDG. The PET measurements were simulated based on the geometry of real clinical scanner (FWHM 4.0mm) with attenuation, scattering and random effects. Poisson noise was added to each generated sinogram bin.

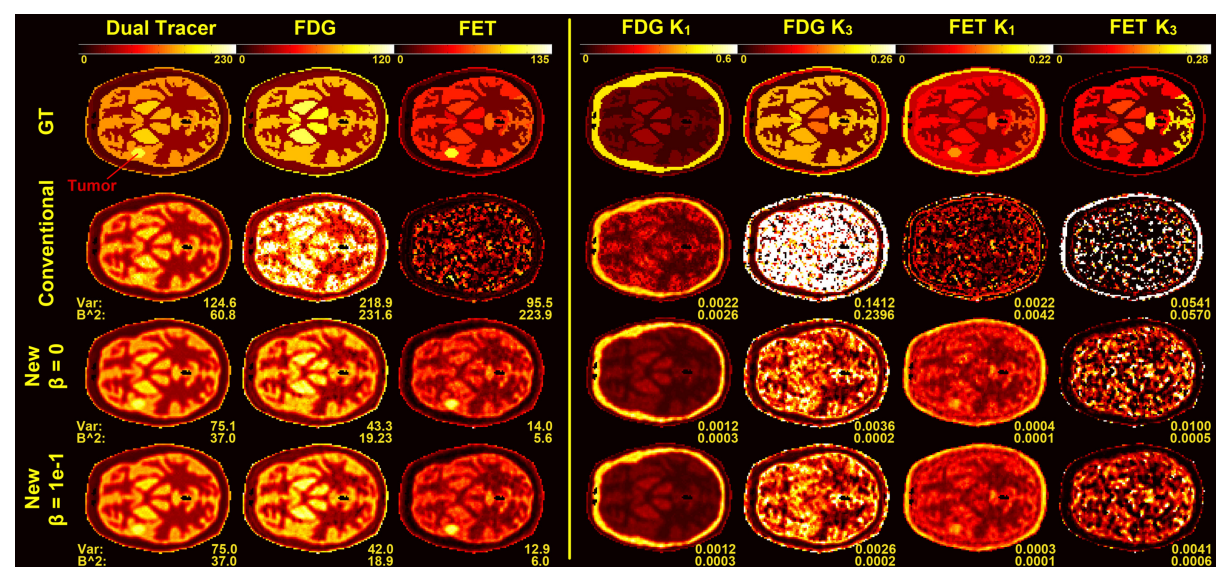

Fig. 1. The results of the separated tracer uptakes and the kinetic parametric images using the conventional and the proposed method on a phantom

Fifty datasets were simulated with different noise realizations. The separation of two tracers were tested with the proposed algorithm without regularization and the conventional indirect method (kinetic modeling after image reconstruction). For both methods, 128 iterations were used for EM reconstruction to keep the balance between bias and noise. For the conventional method, kinetic parameters were estimated using nonlinear least square regression. For fair comparisons, uniform weights were assigned here for the proposed method. Initial values of all parameters in both methods were set to 0.001 for all voxels. The lower and upper bounds were set to 0 and 2.0 respectively. The last frame $(\mathrm{t}=45$ min) of the overlapped and separated tracer uptake maps as well as the parametric images were compared with the ground truth (GT) as shown in Fig. 1 The conventional method was able to recover some voxels of single tracers but it was in general unstable and noisy. With the proposed method, parametric 
images and individual tracer uptakes were well recovered with relative less mean bias $\left(B^{2}\right)$ and variations over all datasets. Tumor is clearly visible in the recovered FET images and no contrast in FDG. Although $k_{3}$ is sensitive to noise, the new method has better result than the conventional method. Overall, the new algorithm has reduced up to $87.8 \%$ variance and $85.7 \%$ bias for the separated tracer uptake maps and up to $97.8 \%$ variance and $98.4 \%$ bias for the parametric images.
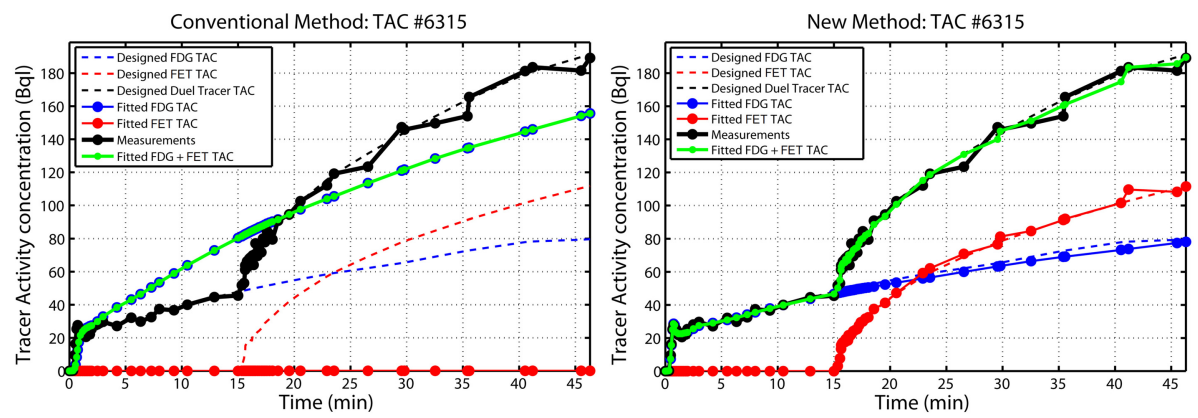

Fig. 2. The fitting results of the conventional (left) and the proposed method (right) for an example TAC in the tumor area

The effect of the regularization term $\beta$ of the proposed algorithm was further evaluated on the phantom. The total square bias and variance of intermediate parameter $\boldsymbol{\nu}$ with $\beta$ from 0 to $4 e-1$ were computed and the variance-bias ${ }^{2}$ curves were plotted in Fig. 3. An optimized $\beta=1 e-1$ was chosen for the tradeoff between bias and variance for $\nu^{F E T}$. The results were shown in Fig. 1 . With the regularization, the variance can be further reduced up to $1.1 \%$ for the separated tracer uptake and $10.1 \%$ for the parametric images.

The conventional method failed to separate the tracer uptake in the tumor area. An example TAC is shown in Fig. 2. The left plot demonstrates the fitting result of the conventional method. It falsely ended up with a zero uptake for FET, which conflicts with the expectation. The right plot shows the result for the proposed algorithm, the temporal information of each tracer were well recovered and reflected the true designed TACs.

We further evaluated the proposed algorithm with the real micro-PET data. Four mice with SUDHL-1 tumor model were scanned for 70 min after injection of approximately $6 \mathrm{MBq}\left[{ }^{18} \mathrm{~F}\right] \mathrm{FDG}$ and $\left[{ }^{18} \mathrm{~F}\right] \mathrm{FLT}$ with an interval of $10 \mathrm{~min}$. The dual-tracer AIF was derived from left ventricle data at early time and 2 venous blood samples in the end of the scan [13]. Two single tracer AIFs were separated by fitting the combined AIF models [14. This lymphoma tumor model has been reported to have higher specificity for FLT [15. Thus the FLT image has higher tumor to muscle contrast than FDG images. The better consistency of the results 

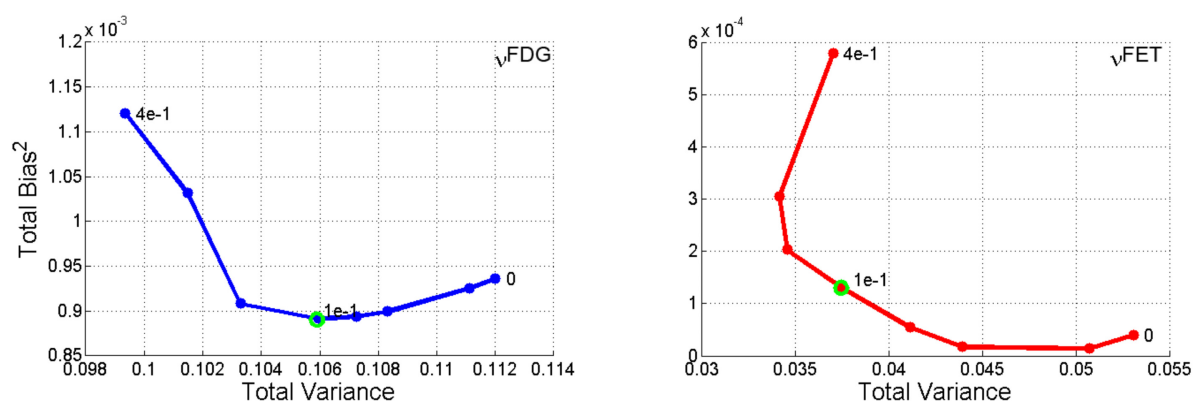

Fig. 3. Total squared bias v.s. variance tradeoff of $\boldsymbol{\nu}$ images

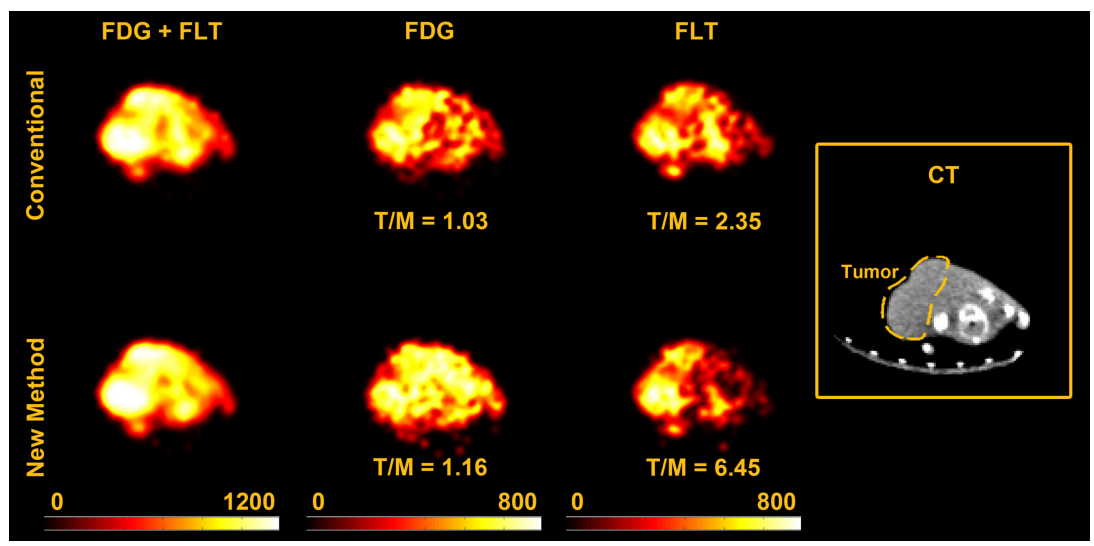

Fig. 4. An example of the results of the conventional and the proposed methods for a mouse with lymphoma tumor model scanned using $\left[{ }^{18} \mathrm{~F}\right] \mathrm{FDG}$ and $\left[{ }^{18} \mathrm{~F}\right] \mathrm{FLT}$ PET

of the new method (an example shown in Fig. 4) with the know properties of the tumor model confirms further that the new method can separate the two tracers better.

\section{Conclusion}

This study proposed a novel direct rapid multi-tracer PET reconstruction algorithm which can robustly retrieve single tracer images from overlapped acquisitions. In particular, we integrated a multi-tracer model with reduced number of parameters into parametric image reconstruction and incorporate EM surrogate functions for the optimization of the penalized log-likelihood. Both phantom and real data has proved that this new algorithm can separate the overlapped signals significantly better than the conventional methods. Further improvements may be done by smoothing noisy TAC for better model fitting concerning low 
measurement statistics of dynamic PET acquisitions. The reliable and less noisy separation of the overlapped PET signal using the proposed method demonstrated a great potential to promote the application of multi-tracer in clinical practice, which could influence the state of the art of the individualized medicine.

Acknowledgement. DFG SFB 824 Z3, BFS, Zhoulei Li (providing tumor cell line), Sybille Reder (technical assistant) and Dr. Guobao Wang (discussion).

\section{References}

1. Kadrmas, D.J., Rust, T.C., Hoffman, J.M.: Single-scan dual-tracer FLT+FDG PET tumor characterization. Phys. Med. Biol. 58, 429-449 (2013)

2. Tian, J., Yang, X., Yu, L., Chen, P., et al.: A multicenter clinical trial on the diagnostic value of dual-tracer PET/CT in pulmonary lesions using 3'-deoxy-3'18F-fluorothymidine and 18F-FDG. J. Nucl. Med. 49, 186-194 (2008)

3. Andreyev, A., Celler, A.: Dual-isotope PET using positron-gamma emitters. Phys. Med. Biol. 56, 4539-4556 (2011)

4. Kadrmas, D., Rust, T.: Feasibility of rapid multitracer PET tumor imaging. IEEE Trans. Nucl. Sci. 52, 1341-1347 (2005)

5. Verhaeghe, J., Reader, A.J.: Accelerated PET water activation acquisition with signal separation methodology. Med. Phys. 40 (2013)

6. Gao, F., Liu, H., Jian, Y., Shi, P.: Dynamic dual-tracer PET reconstruction. In: Prince, J.L., Pham, D.L., Myers, K.J. (eds.) IPMI 2009. LNCS, vol. 5636, pp. 38-49. Springer, Heidelberg (2009)

7. Oktay, M., Kadrmas, D.: Reduced parameter space formulations for fast and robust kinetic modeling. SNM 53, 2292 (2012)

8. Matthews, J., Bailey, D., Price, P., et al.: The direct calculation of parametric images from dynamic PET data using maximum-likelihood iterative reconstruction. Phys. Med. Biol. 42, 1155-1173 (1997)

9. Yan, J., Planeta-Wilson, B., Gallezot, J.D., et al.: Initial evaluation of direct 4D parametric reconstruction with human PET data. IEEE NSS 1, 2503-2506 (2009)

10. De Pierro, A.: A modified EM algorithm for penalized likelihood estimation in emission tomography. IEEE Trans. Med. Imaging 14, 132-137 (1995)

11. Wang, G., Qi, J.: An optimization transfer algorithm for nonlinear parametric image reconstruction from dynamic PET data. IEEE Trans. Med. Imaging 31, 1977-1988 (2012)

12. Gunn, R.N., Gunn, S.R., Cunningham, V.J.: Positron emission tomography compartmental models. J. Cereb. Blood. Flow. Metab. 21, 635-652 (2001)

13. Ferl, G.Z., Zhang, X., Wu, H.M., et al.: Estimation of the 18F-FDG input function in mice by use of dynamic small-animal PET and minimal blood sample data. J. Nucl. Med. 48, 2037-2045 (2007)

14. Feng, D., Huang, S.C., Wang, X.: Models for computer simulation studies of input functions for tracer kinetic modeling with PET. Int. J. Biomed. Comput. 32, 95110 (1993)

15. Li, Z., Graf, N., Herrmann, K., Junger, et al.: FLT-PET is superior to FDG-PET for very early response prediction in NPM-ALK-positive lymphoma treated with targeted therapy. Cancer. Res. 72, 5014-5024 (2012) 